\title{
In vitro Susceptibilities of Methicillin- Susceptible and Resistant Staphylococci to Traditional Antibiotics Compared to a Novel Fluoroquinolone
}

\author{
Kenneth C. Fan, James Lin, Nicolas A. Yannuzzi, Hasenin Al-Khersan, Nimesh A. Patel, Jorge Maestre-Mesa, \\ Mustafa Zaidi, Darlene Miller and Harry W. Flynn Jr* (i)
}

\begin{abstract}
Background: To assess the in-vitro efficacy of delafloxacin, a new fourth generation fluoroquinolone, against Staphylococcus vitreous isolates from patients with clinically diagnosed endophthalmitis. This is the first investigation of delafloxacin in ocular tissues.
\end{abstract}

Methods: Intravitreal isolates of culture-proven S. aureus and S. epidermidis were identified between 2014 and 2018. Minimum inhibitor concentrations (MIC) were determined using ETEST strips. The antibiotic susceptibilities were tested against a panel of drugs including glycopeptides such as vancomycin, as well as traditional and newer fluoroquinolones (levofloxacin, moxifloxacin, and delafloxacin).

Results: Of 45 total isolates identified between 2014 and 2018, 13\% (6) were methicillin-resistant S. aureus (MRSA), 9\% (4) were methicillin-sensitive S. aureus (MSSA), 53\% (24) were methicillin-resistant S. epidermidis (MRSE), and 24\% (11) were methicillin-sensitive S. epidermidis (MSSE). Among the fluoroquinolones, resistance rates were $61 \%$ for levofloxacin, 50\% for moxifloxacin, and 12\% for delafloxacin. Inter-class comparisons between delafloxacin and the two other fluoroquinolones demonstrated higher Gram-positive susceptibility to delafloxacin $(p<0.01)$. MIC90 values were lowest for delafloxacin $(1.0 \mu \mathrm{g} / \mathrm{mL})$ compared to levofloxacin $(8.0 \mu \mathrm{g} / \mathrm{mL})$ and moxifloxacin $(8.0 \mu \mathrm{g} / \mathrm{mL})$. Vancomycin was 100\% effective against all isolates with MIC90 value of $0.75 \mu \mathrm{g} / \mathrm{mL}$.

Conclusion: Compared to levofloxacin and moxifloxacin, the newer fluoroquinolone delafloxacin demonstrated the lowest MICs values and lowest rates of resistance for Gram-positive in-vitro S. epidermidis and S. aureus vitreous isolates.

Keywords: Antibiotic resistance, Endophthalmitis, Fluoroquinolone, Minimum inhibitory concentration, Staphylococcus aureus, Staphylococcus epidermidis, Vitreous

\section{Introduction}

Endophthalmitis is a devastating intraocular condition caused by a variety of different microbiological organisms. The most common category of exogenous endophthalmitis is acute-onset post-operative, comprising 40-80\% of all causes of endophthalmitis [1]. Other less common etiologies include post-injection and post-traumatic endophthalmitis $[1,2]$. Across these mechanisms of infection, the most common causative organism is coagulase-negative

\footnotetext{
* Correspondence: hflynn@med.miami.edu

Department of Ophthalmology, Bascom Palmer Eye Institute, Miller School of Medicine, University of Miami, Miami, FL, USA
}

Staphylococcus (CoNS) [1-6]. Prior studies have established high rates of antibiotic resistance in Gram-positive endophthalmitis vitreous isolates, with resistance to fluoroquinolones including ciprofloxacin and levofloxacin as high as $41 \%$ and $56 \%$, respectively $[5,7,8]$. Furthermore, trends towards increasing drug resistance of Gram-positive vitreous isolates have been demonstrated [5, 8-11]. In light of these data, the development and investigation of newer, potentially more effective antibiotics for endophthalmitis is clinically important.

Delafloxacin is a new broad-spectrum fluoroquinolone that was approved by the FDA in June 2017 for oral and 
intravenous use in the treatment of acute bacterial skin and skin structure infections (ABSSSIs) after demonstrating non-inferiority to vancomycin and aztreonam [12]. To date, there are no reports demonstrating the effect of delafloxacin in intraocular tissue infections either in vivo or in vitro. The purpose of the current study is to investigate the possible role that delafloxacin may play in treating infectious endophthalmitis.

\section{Methods}

Institutional review board approval was obtained from the University of Miami Miller School of Medicine Sciences Subcommittee for the Protection of Human Subjects and the research followed the tenets of the Declaration of Helsinski (IRB Protocol Study ID \#20120897). The Ocular Microbiology Department database was searched to identify nonconsecutive positive intravitreal isolates of culture-proven Staphylococcus aureus and Staphylococcus epidermidis organisms between January 1, 2014 and December 31, 2018. Records from the microbiology department were reviewed to confirm isolates and identify antimicrobial susceptibilities.

Isolates were cultured using standard microbiological procedures. Vitreous cultures were obtained at the time of vitreous tap or vitrectomy in patients with endophthalmitis. For vitreous tap samples, fluid was directly cultured onto 5\% sheep blood and chocolate agar culture media. For vitrectomy samples, $30-50 \mathrm{~mL}$ of vitreous washings were filtered using a $0.45-\mu \mathrm{m}$ filter, which were divided into segments and plated onto culture media, including 5\% sheep blood and chocolate agar. Blood and chocolate agar plates underwent incubation at $35^{\circ} \mathrm{C}$ for up to 2 weeks. Additional culture media, including thioglycollate broth, was submitted at the discretion of the ophthalmologist performing the culture.

A standard inoculum $\left(1 \times 10^{8} \mathrm{CFU} / \mathrm{mL}\right)$ for each isolate was placed on Mueller-Hinton agar. Minimum inhibitor concentrations (MIC) were determined using ETEST strips (bioMérieux, Marcy l'Etoile, France) placed according to manufacturer's instructions, and susceptibilities were based on breakpoints from Clinical \& Laboratory Standards Institute (CLSI) guidelines (see Fig. 1). MIC90 was calculated based on the concentration at which growth of all organisms were inhibited. Plates were incubated in a non- $\mathrm{CO}_{2}$ incubator and read after $18-24 \mathrm{~h}$. The antibiotic susceptibilities were tested against vancomycin as well as traditional and newer fluoroquinolones (levofloxacin, moxifloxacin, and delafloxacin).

Organisms were graded as either susceptible or resistant to each antibiotic. Cases of indeterminate resistance were classified as antibiotic resistant. Pearson chi-squared testing was used to compare relative antibiotic susceptibility among each of the fluoroquinolone antibiotics. A $p$ value $<0.05$ was considered statistically significant. Statistical analysis was carried out using Stata 15 (StataCorp, College Station, TX).

\section{Results}

Of 45 total isolates identified between 2014 and 2018, 13\%

(6) were methicillin-resistant S. aureus (MRSA), 9\% (4) were methicillin-sensitive S. aureus (MSSA), 53\% (24) were methicillin-resistant S. epidermidis (MRSE), and 24\% (11) were methicillin-sensitive S. epidermidis (MSSE, see Table 1). Among the fluoroquinolones, resistance rates across all organisms were $60 \%$ for levofloxacin, $50 \%$ for moxifloxacin, and $12 \%$ for delafloxacin (see Table 1). Direct comparisons between delafloxacin and moxifloxacin across all Staphylococcus isolates demonstrated higher susceptibilities for delafloxacin $(p<0.01)$. Likewise, direct comparisons between delafloxacin and levofloxacin across all Staphylococcus isolates demonstrated higher susceptibilities for delafloxacin $(p<0.01)$. Vancomycin was effective against all isolates. For MSSA, vitreous isolate samples demonstrated a $50 \%$ rate of resistance for levofloxacin, $50 \%$ for moxifloxacin, and 0\% for delafloxacin. MSSE displayed a similar trend, with $60 \%$ resistance for levofloxacin, $55 \%$ for moxifloxacin, and $27 \%$ for delafloxacin. For isolates that were resistant to methicillin (MRSA and MRSE), resistance rates increased significantly to $83 \%$, $83 \%$, and $33 \%$ rates of resistance against levofloxacin, moxifloxacin, and delafloxacin respectively, and for MRSE, $54 \%, 42 \%$, and $25 \%$ rates of resistance against levofloxacin, moxifloxacin, and delafloxacin respectively (see Table 1).

MIC90 values were lowest for delafloxacin $(1.0 \mu \mathrm{g} / \mathrm{mL})$ compared to levofloxacin $(8.0 \mu \mathrm{g} / \mathrm{mL})$ and moxifloxacin $(8.0 \mu \mathrm{g} / \mathrm{mL}$, see Table 1$)$. The MIC90 for vancomycin was $0.75 \mu \mathrm{g} / \mathrm{mL}$.

\section{Discussion}

The most common microbes causing exogenous endophthalmitis are Gram-positive cocci, namely CoNS as well as $S$. aureus $[1,2,5,13]$. In the Endophthalmitis Vitrectomy Study as well as some more recent studies, CoNS and $S$. aureus have remained at similar post-operative prevalence rates following cataract surgery through the decades (approximately $60 \%$ and $10 \%$, respectively) $[6,10,13]$. Occurring at a rate of $0.056 \%$, endophthalmitis after intravitreal injection is the second most common category of endophthalmitis, with CoNS comprising 38\% of cases, followed by Streptococcus species and S. aureus [2].

Current empiric treatment of suspected bacterial endophthalmitis typically begins with intravitreal vancomycin and ceftazidime $[1,6,14-16]$. Regarding fluoroquinolones, intracameral and systemic treatment has gained interest in light of the favorable safety profile and low MIC levels of some agents like moxifloxacin $[15,17]$. However, steadily increasing rates of antibiotic resistance to fluoroquinolones among CoNS have been reported (56\% nonsusceptibility for ciprofloxacin and levofloxacin, $57 \%$ for moxifloxacin) [7], with these resistant microbes conferring poor visual outcomes [18]. 


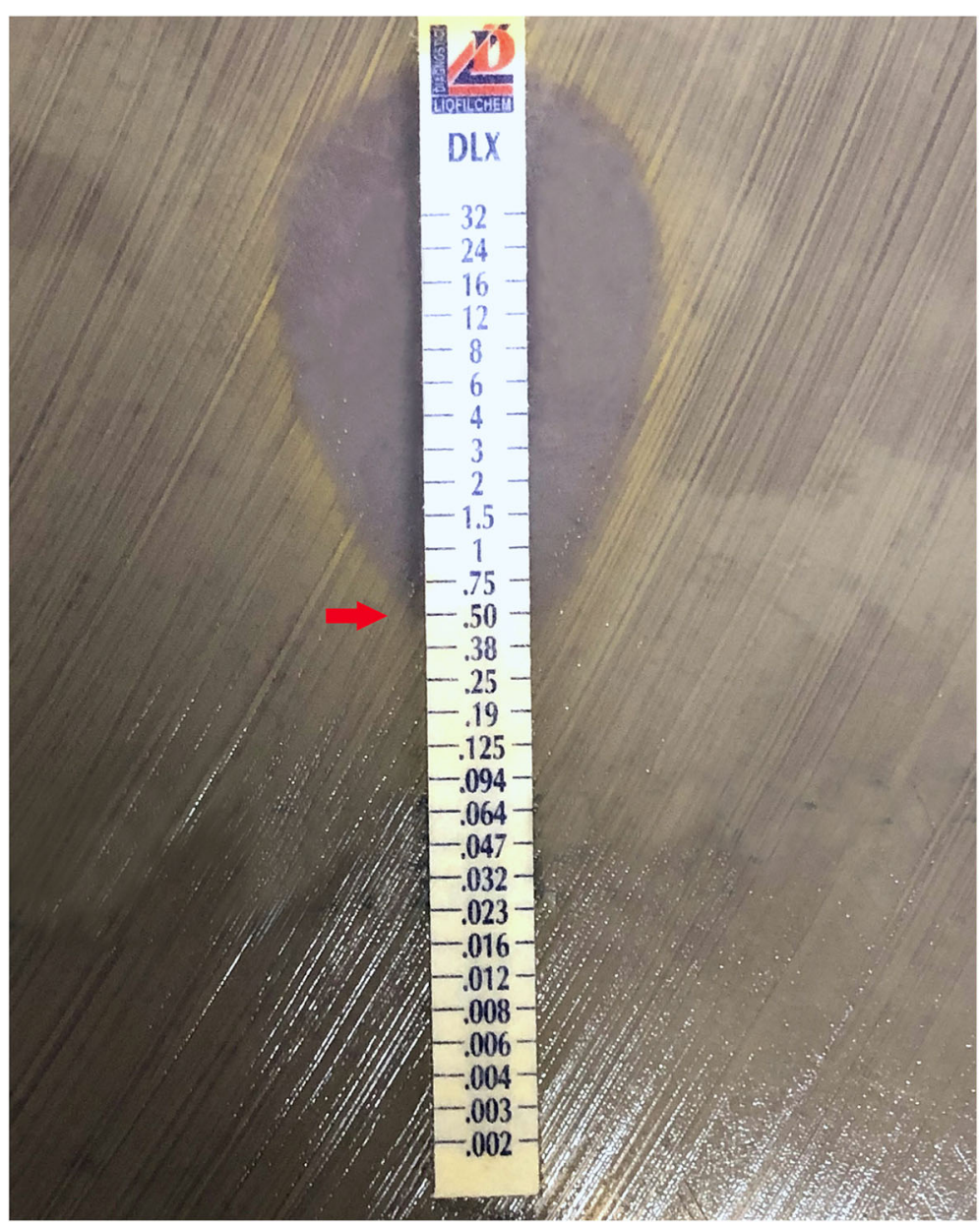

Fig. 1 Growth of Gram-positive organisms from intravitreal endophthalmitis isolates on Mueller-Hinton agar with ETEST strip testing for antibiotic susceptibilities and minimum inhibitory concentrations. The drug is eluted in immediate proximity to the plastic carrier, creating a gradient of drug concentrations to measure the minimum concentration required for the inhibition of growth. This figure represents inhibition of growth (red arrow), a minimum concentration of $0.5 \mu \mathrm{g} / \mathrm{ml}$.

The current study examines the in-vitro efficacy of a new fourth-generation fluoroquinolone, delafloxacin, for S. epidermidis and $S$. aureus endophthalmitis vitreous isolates in a single, tertiary care institution over a 4-year period. Delafloxacin is a promising new antibiotic that targets bacterial topoisomerase IV and DNA gyrase and demonstrates activity against Gram-positive bacteria including MRSA and Gram- negative bacteria such as Pseudomonas aeruginosa ( $P$. aeruginosa) [15]. In vivo pharmacokinetic and pharmacodynamic studies have demonstrated rapid oral absorption of delafloxacin, high bioavailability (60-70\%), and renal excretion [19]. Delafloxacin is a concentration-dependent fluoroquinolone, exhibiting in vivo MICs of $\leq 1 \mathrm{mg} / \mathrm{L}$ against $P$. aeruginosa and $0.25 \mathrm{mg} / \mathrm{L}$ for Klebsiella pneumoniae, which predicts

Table 1 Antimicrobial activities of delafloxacin, levofloxacin, and moxifloxacin tested against intravitreal Staphylococcus isolates

\begin{tabular}{|c|c|c|c|c|c|c|c|c|c|c|}
\hline \multirow[t]{2}{*}{ Organism } & \multicolumn{10}{|c|}{ Rates of resistance } \\
\hline & All isolates & $\mathrm{MIC}{ }^{\mathrm{a}}$ & MSSA $^{b}$ & MIC90 & MSSE $^{c}$ & MIC90 & MRSA $^{d}$ & MIC90 & MRSE $^{e}$ & MIC90 \\
\hline Levofloxacin & $60 \%(26 / 43)$ & 8.0 & $50 \%(2 / 4)$ & -- & $60 \%(6 / 10)$ & 8.0 & $83 \%(5 / 6)$ & -- & $54 \%(13 / 24)$ & 8.0 \\
\hline Moxifloxacin & $50 \%(22 / 44)$ & 8.0 & $50 \%(2 / 4)$ & -- & $55 \%(6 / 11)$ & 8.0 & $83 \%(5 / 6)$ & -- & $54 \%(13 / 24)$ & 8.0 \\
\hline Delafloxacin & $12 \%(12 / 43)$ & 1.0 & $0 \%(0 / 4)$ & -- & $27 \%(3 / 11)$ & 0.8 & $33 \%(2 / 6)$ & -- & $25 \%(6 / 24)$ & 0.8 \\
\hline
\end{tabular}

${ }^{2}$ MIC90, minimum inhibitory concentration at which growth of $90 \%$ of organisms is inhibited, expressed in micrograms per milliliter ( $\left.\mu \mathrm{g} / \mathrm{ml}\right)$, which were only calculated for microbes with more than 10 isolates

${ }^{\mathrm{b}} \mathrm{MSSA}$, methicillin-sensitive $S$. aureus

'MRSA,methicillin-resistant S. aureus

${ }^{\mathrm{d} M S S E}$, methicillin-sensitive S. epidermidis

${ }^{M}$ MRSE, methicillin-resistant S. epidermidis 
efficacy against $75 \%$ and $78 \%$ of strains respectively [19-21]. In vitro studies have shown MIC levels for delafloxacin to be 3-5 times lower than other fluoroquinolones against Gram-positive microbes [21]. Additionally, multiple groups have shown its higher in vitro efficacy against Gram-positive organisms compared to other in-class agents [22, 23].

The findings in the current study demonstrate that delafloxacin is a more effective fluoroquinolone than either levofloxacin or moxifloxacin for in-vitro Gram-positive vitreous isolates, with lower nonsusceptibility rates for both S. epidermidis and S. aureus. This has been demonstrated in previous studies for ABSSSIs as well and now is confirmed for ocular tissues [23]. Additionally, for methicillinresistant strains of $S$. epidermidis and $S$. aureus, rates of resistance were also comparatively lower for delafloxacin. MIC90 levels reported were significantly lower for delafloxacin $(1.0 \mu \mathrm{g} / \mathrm{mL})$ compared to $8.0 \mu \mathrm{g} / \mathrm{mL}$ for both levofloxacin and moxifloxacin, indicating that lower concentrations are required to reach therapeutic levels against vitreous isolates in vitro. This lower MIC represents an 8-fold decrease in concentration required for inhibition of growth compared to other fluoroquinolones, which had been previously reported as 3 - to 5 -fold lower in vitro [21].

Similar to prior studies, vancomycin was effective against all 45 isolates in this study, and it remains one of the most effective empiric treatments for endophthalmitis $[5,6,9,10,24]$. However, delafloxacin has the benefit of wider microbial coverage including Gram-negative organisms like $P$. aeruginosa, anaerobes, and atypical infections $[25,26]$. Systemically, safety studies have not shown any QTc prolongation or evidence of phototoxicity often seen in other fluoroquinolones; however, some risk of tendonitis still exists [21]. Further investigations on the bioavailability, intraocular toxicity, and penetration of ocular tissues are required.

The limitations of this study include the analysis of only CoNS and $S$. aureus organisms, the retrospective design of the study, and lack of an intraocular formulary for delafloxacin. Expanding the analysis to both Grampositive and Gram-negative organisms may lend further utility in determining the role of delafloxacin in endophthalmitis. Due to a retrospective design, stratification by post-operative, post-injection, post-traumatic, or other causes was not possible. ETEST strips were used for determination of MIC, which allowed only for close but still accurate approximation. Lastly, delafloxacin has only been approved in oral and intravenous formulations, and safety and pharmacokinetics studies have not been performed in intraocular tissues.

\section{Conclusion}

Delafloxacin is an exciting new drug that demonstrates potential utility in endophthalmitis for Staphylococcus vitreous isolates due to low MIC levels and low comparative rates of nonsusceptibility even for methicillin-resistant organisms. Compared to levofloxacin and moxifloxacin, the newer fluoroquinolone delafloxacin demonstrated the lowest MICs values and lowest rates of resistance for Gram-positive in-vitro S. epidermidis and S. aureus vitreous isolates. The current landscape of rising antibiotic resistance makes the investigation of newer, more efficacious therapies important for improving clinical outcomes. Delafloxacin is an expensive drug, however, costing approximately $\$ 675$ for a 5 -day course of systemic treatment (compared to $\$ 51$ for moxifloxacin) [15]. Therefore, future investigations on cost utility of the drug as well as widerscale prospective studies will help define its promising role in the treatment of endophthalmitis.

\section{Abbreviations}

ABSSSI: Acute bacterial skin and skin structure infections; CLSI: Clinical \& Laboratory Standards Institute; CoNS: Coagulase-negative Staphylococcus; MIC: Minimum inhibitory concentration; MRSA : Methicillin-resistant

Staphylococcus aureus; MRSE: Methicillin-resistant Staphylococcus epidermidis; MSSA: Methicillin-sensitive Staphylococcus aureus; MSSE: Methicillin-sensitive

Staphylococcus epidermidis

\section{Acknowledgements}

Not applicable

\section{Authors' contributions}

$\mathrm{KF}, \mathrm{DM}$, and HF coordinated the study design, analyzed and interpreted data, and were involved in all the stages of the manuscript preparation. JM and MZ collected and analyzed the data. JL, NAY, NAP, and HA were involved in the statistical analysis and manuscript preparation. All authors read and approved the final manuscript.

\section{Funding}

This study was supported by NIH Center Core Grant P30EY014801, Research to Prevent Blindness Unrestricted Grant.

\section{Availability of data and materials}

The datasets used and analyzed during the current study are available from the corresponding author upon reasonable request.

\section{Ethics approval and consent to participate}

Institutional review board approval was obtained from the University of Miami Miller School of Medicine Sciences Subcommittee for the Protection of Human Subjects; and the research followed the tenets of the Declaration of Helsinski (IRB Protocol Study ID no. 20120897).

\section{Consent for publication}

Not applicable

\section{Competing interests}

The authors KCF, JL, NAY, HA, NAP, JM, MZ, DM, and HWF have no financial or proprietary interests in the completion of this study.

Received: 7 September 2019 Accepted: 3 February 2020

Published online: 27 February 2020

References

1. Durand ML (2017) Bacterial and fungal endophthalmitis. Clin Microbiol Rev. 30(3):597-613

2. Fileta JB, Scott IU, Flynn HW Jr (2014) Meta-analysis of infectious endophthalmitis after intravitreal injection of anti-vascular endothelial growth factor agents. Ophthalmic Surg Lasers Imaging Retina. 45(2): 143-149

3. Andreoli CM, Andreoli MT, Kloek CE, Ahuero AE, Vavvas D, Durand ML (2009) Low rate of endophthalmitis in a large series of open globe injuries. Am J Ophthalmol. 147(4):601-608 e2 
4. McCannel CA (2011) Meta-analysis of endophthalmitis after intravitreal injection of anti-vascular endothelial growth factor agents: causative organisms and possible prevention strategies. Retina. 31(4):654-661

5. Benz MS, Scott IU, Flynn HW Jr, Unonius N, Miller D (2004) Endophthalmitis isolates and antibiotic sensitivities: a 6-year review of culture-proven cases. Am J Ophthalmol. 137(1):38-42

6. Yannuzzi NA, Si N, Relhan N, Kuriyan AE, Albini TA, Berrocal AM, Davis JL, Smiddy WE, Townsend J, Miller D, Flynn HW Jr (2017) Endophthalmitis after clear corneal cataract surgery: outcomes over two decades. Am J Ophthalmol. 174:155-159

7. Stringham JD, Relhan N, Miller D, Flynn HW Jr (2017) Trends in fluoroquinolone nonsusceptibility among coagulase-negative Staphylococcus isolates causing endophthalmitis, 1995-2016. JAMA Ophthalmol. 135(7):814-815

8. Schimel AM, Miller D, Flynn HW (2012) Evolving fluoroquinolone resistance among coagulase-negative Staphylococcus isolates causing endophthalmitis. Arch Ophthalmol. 130(12):1617-1618

9. Gentile RC, Shukla S, Shah M, Ritterband DC, Engelbert M, Davis A, Hu DN (2014) Microbiological spectrum and antibiotic sensitivity in endophthalmitis: a 25-year review. Ophthalmology. 121(8):1634-1642

10. Recchia FM, Busbee BG, Pearlman RB, Carvalho-Recchia CA, Ho AC (2005) Changing trends in the microbiologic aspects of postcataract endophthalmitis. Arch Ophthalmol. 123(3):341-346

11. Deramo VA, Lai JC, Fastenberg DM, Udell IJ (2006) Acute endophthalmitis in eyes treated prophylactically with gatifloxacin and moxifloxacin. Am J Ophthalmol. 142(5):721-725

12. Bassetti M, Righi E, Pecori D, Tillotson G (2018) Delafloxacin: an improved fluoroquinolone developed through advanced molecular engineering. Future Microbiol. 13:1081-1094

13. Endophthalmitis Vitrectomy Study Group (1995) Results of the endophthalmitis vitrectomy study. A randomized trial of immediate vitrectomy and of intravenous antibiotics for the treatment of postoperative bacterial endophthalmitis. Arch Ophthalmol 113(12):1479-1496

14. Aaberg TM Jr, Flynn HW Jr, Murray TG (1994) Intraocular ceftazidime as an alternative to the aminoglycosides in the treatment of endophthalmitis. Arch Ophthalmol. 112(1):18-19

15. Clarke B, Williamson TH, Gini G, Gupta B (2018) Management of bacterial postoperative endophthalmitis and the role of vitrectomy. Surv Ophthalmol. 63(5):677-693

16. Liu C, Ji J, Li S, Wang Z, Tang L, Cao W, Sun X (2017) Microbiological isolates and antibiotic susceptibilities: a 10-year review of culture-proven endophthalmitis cases. Curr Eye Res. 42(3):443-447

17. Vedantham V, Lalitha P, Velpandian T, Ghose S, Mahalakshmi R, Ramasamy K (2006) Vitreous and aqueous penetration of orally administered moxifloxacin in humans. Eye (Lond). 20(11):1273-1278

18. Chiquet C, Maurin M, Altayrac J, Aptel F, Boisset S, Vandenesch F, Cornut PL, Romanet JP, Gain P, Carricajo A (2015) Correlation between clinical data and antibiotic resistance in coagulase-negative Staphylococcus species isolated from 68 patients with acute post-cataract endophthalmitis. Clin Microbiol Infect 21(6):592 e1-8

19. Shiu J, Ting G, Kiang TK (2019) Clinical pharmacokinetics and pharmacodynamics of delafloxacin. Eur J Drug Metab Pharmacokinet. 44(3):305-317

20. Zhao M, Lepak AJ, Marchillo K, Andes DR (2019) In vivo pharmacodynamic target determination for delafloxacin against Klebsiella pneumoniae and Pseudomonas aeruginosa in the neutropenic murine pneumonia model. Antimicrob Agents Chemother. 63(10)

21. Jorgensen SCJ, Mercuro NJ, Davis SL, Rybak MJ (2018) Delafloxacin: place in therapy and review of microbiologic, clinical and pharmacologic properties. Infect Dis Ther. 7(2):197-217

22. O'Riordan W, Mc Manus A, Teras J, Poromanski I, Cruz-Saldariagga M, Quintas M, Lawrence L, Liang S, Cammarata S, Group PS (2018) A comparison of the efficacy and safety of intravenous followed by oral delafloxacin with vancomycin plus aztreonam for the treatment of acute bacterial skin and skin structure infections: a phase 3, multinational, doubleblind, randomized study. Clin Infect Dis. 67(5):657-666

23. McCurdy S, Lawrence L, Quintas M, Woosley L, Flamm R, Tseng C, Cammarata S (2017) In vitro activity of delafloxacin and microbiological response against fluoroquinolone-susceptible and nonsusceptible Staphylococcus aureus isolates from two phase 3 studies of acute bacterial skin and skin structure infections. Antimicrob Agents Chemother. 61(9)
24. Teng YT, Teng MC, Kuo HK, Fang PC, Wu PC, Chen CH, Kuo MT, Yang IH, Chen YJ (2017) Isolates and antibiotic susceptibilities of endophthalmitis in postcataract surgery: a 12-year review of culture-proven cases. Int Ophthalmol. 37(3):513-518

25. Nilius AM, Shen LL, Hensey-Rudloff D, Almer LS, Beyer JM, Balli DJ, Cai Y, Flamm RK (2003) In vitro antibacterial potency and spectrum of ABT-492, a new fluoroquinolone. Antimicrob Agents Chemother. 47(10):3260-3269

26. Saravolatz LD, Stein GE (2019) Delafloxacin: a new anti-methicillin-resistant Staphylococcus aureus fluoroquinolone. Clin Infect Dis. 68(6):1058-1062

\section{Publisher's Note}

Springer Nature remains neutral with regard to jurisdictional claims in published maps and institutional affiliations.

\section{Submit your manuscript to a SpringerOpen ${ }^{\circ}$ journal and benefit from:}

- Convenient online submission

- Rigorous peer review

- Open access: articles freely available online

- High visibility within the field

- Retaining the copyright to your article

Submit your next manuscript at $\boldsymbol{\nabla}$ springeropen.com 